\title{
Incomprehensible treatment of retinoblastoma with high doses of vitamin $\mathrm{C}$
}

\author{
Tomcikova $\mathrm{D}^{1}$, Gerinec $\mathrm{A}^{1}$, Busanyova $\mathrm{B}^{1}$, Husakova $\mathrm{K}^{2}$, Kuniak $\mathrm{M}^{3}$, Autrata $\mathrm{R}^{4}$ \\ Pediatric Ophthalmology Department, Children’s University Hospital, Bratislava, Slovakia. \\ dtomcikova@gmail.com
}

\section{ABSTRACT}

PURPOSES: To inform about a case of neglected retinoblastoma that was left untreated for more than 3 years by parents. During this time period the local finding worsened from endophytic retinoblastoma group $B$ according IIRC (ABC classification) to extraorbital propagation.

BACKGROUND: Retinoblastoma is the most common intraocular tumor in childhood, that occurs approximately in 1 : 15-20 000 births worldwide. In European region cases of extraocular propagation are very infrequent. Extraorbital propagation is extremely rare in middle and high income countries.

METHODS: We report the preoperative ophthalmological findings, MRI imaging, treatment methods and postoperative results of this case.

RESULTS: After initial dose of six courses of chemotherapy patient underwent surgery (orbital exenteration). In postoperative period patient received two more courses of chemotherapy. In spite of progressed stage of the disease, we obtained good results with our therapy.

CONCLUSIONS: We suppose that good treatment results, in spite of extraordinary long lag interval and hopeless pretreatment condition, caused by alternative therapy with high doses vitamin $\mathrm{C}$ and no protein intake, were caused by therapeutic naïve retinoblastoma with an absence of RB1 gene mutation (Fig. 6, Ref. 7). Text in PDF www.elis.sk.

KEY WORDS: extraorbital retinoblastoma, treatment, high doses of vitamin C, exenteration, RB1 gene.

\section{Introduction}

Retinoblastoma is the most common intraocular tumor in childhood, which occurs approximately in 1: 15-20 000 live births worldwide. In European region, contrary to the low income countries, extraocular propagation is very infrequent. Extraorbital propagation is extremely rare in middle and high income countries. In Slovakia it is the first case of extraorbital propagation of retinoblastoma.

\section{Materials and methods}

The patient was referred to our Pediatric Ophthalmology Department in May 2013 at the age of 9 months. It was a Caucasian male. Family history was negative for retinoblastoma, the social conditions were good in the family. By fundus examination under general anesthesia we observed a solitary tumor. It was located on posterior pole, in macular region, near, but not involving the

'Pediatric Ophthalmology Department, Children's University Hospital, Bratislava, Slovakia, ${ }^{2}$ Department of Pediatric Hematology and Oncology, Children's University Hospital, Bratislava, Slovakia, ${ }^{3}$ Department of Neurosurgery, University Hospital, Bratislava, Slovakia, and ${ }^{4}$ Pediatric Ophthalmology Department, University Hospital, Brno, Czech Republic Address for correspondence: D. Tomčíková, Pediatric Ophthalmology Department, Children's University Hospital, SK-833 01 Bratislava, Slovakia. Phone: +421.905463981 optic disc. The tumor was 5PD in diameter and $5 \mathrm{sphD}$ in elevation. Endophytic retinoblastoma group B according to IIRC (ABC classification) was diagnosed, which has very favorable prognosis according to globe salvation (Fig. 1). Parents refused therapy in our country and went abroad. We didn't know that they have chosen alternative therapy with no proteins intake and high doses of vitamin $\mathrm{C}$ supplementation. The local finding worsened to painful secondary glaucoma, because of tumor growth. The eye was enucleated in 2015 (in Hungary) with microscopic evidence of tu-

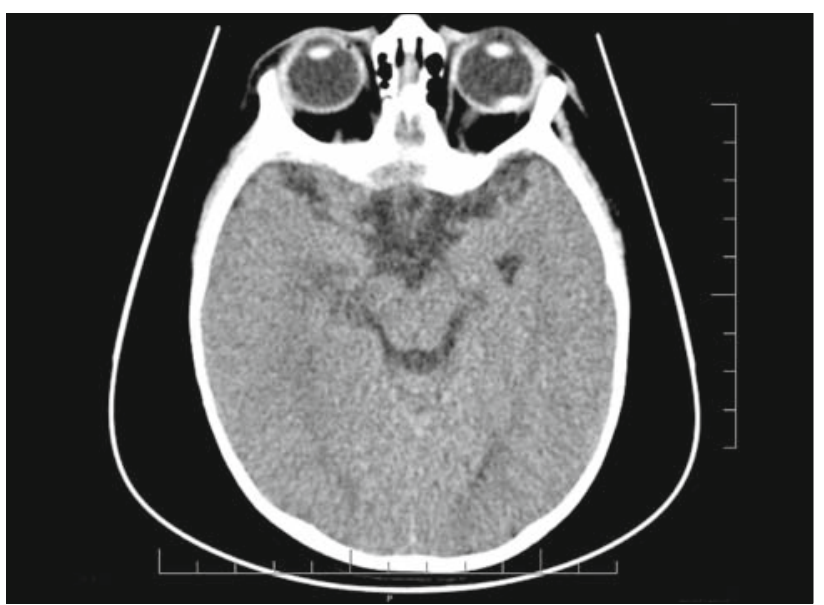

Fig. 1. Patient D.S. May 2013: Intraocular retinoblastoma gr. B (IIRC). 


\section{3-515}

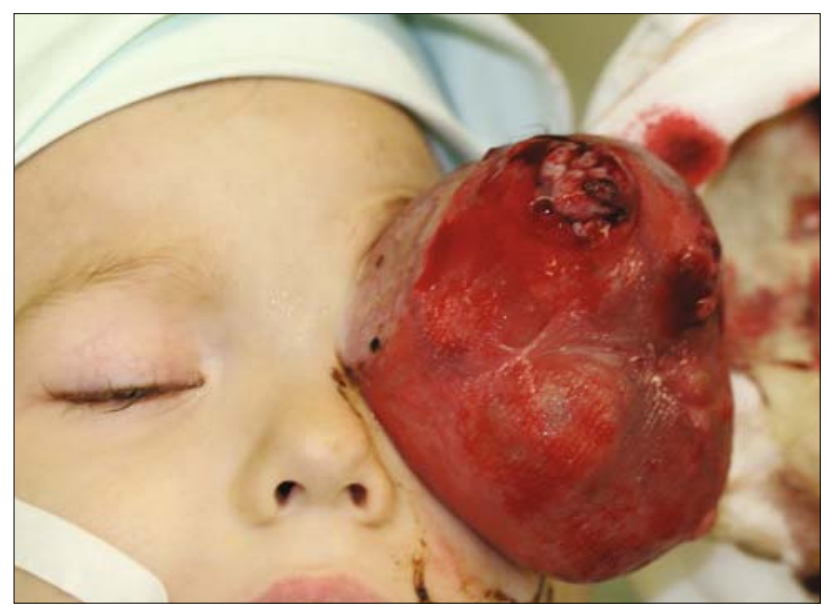

Fig. 2. Patient D.S., October 2016, Extraorbital mass of retinoblastoma.

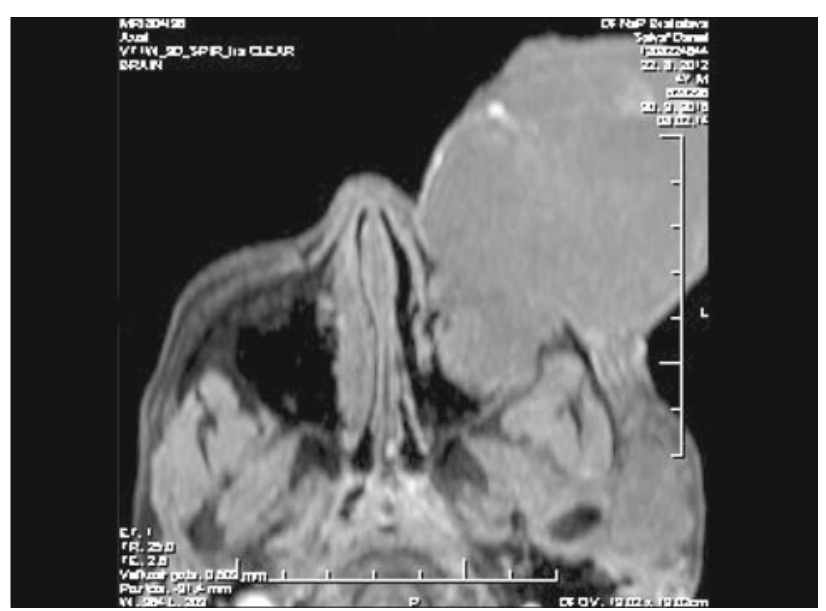

Fig. 3. MRI, October 2016, Extraorbital mass of retinoblastoma.

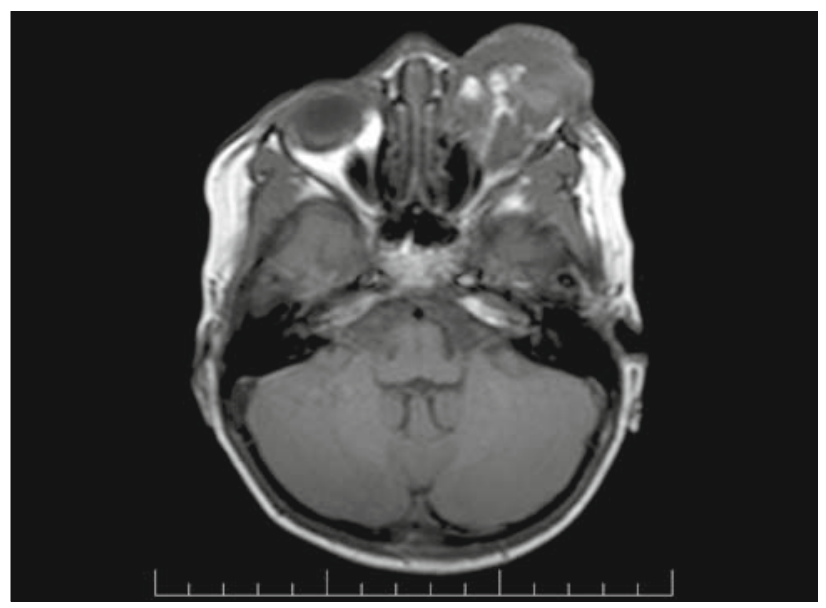

Fig. 4. MRI, February 2017, tumor reduction after 6 courses of CHT.

mor invasion at surgical section of the optic nerve. Chemotherapy was recommended, but the parents refused it and continued with alternative therapy. They returned to our department in October

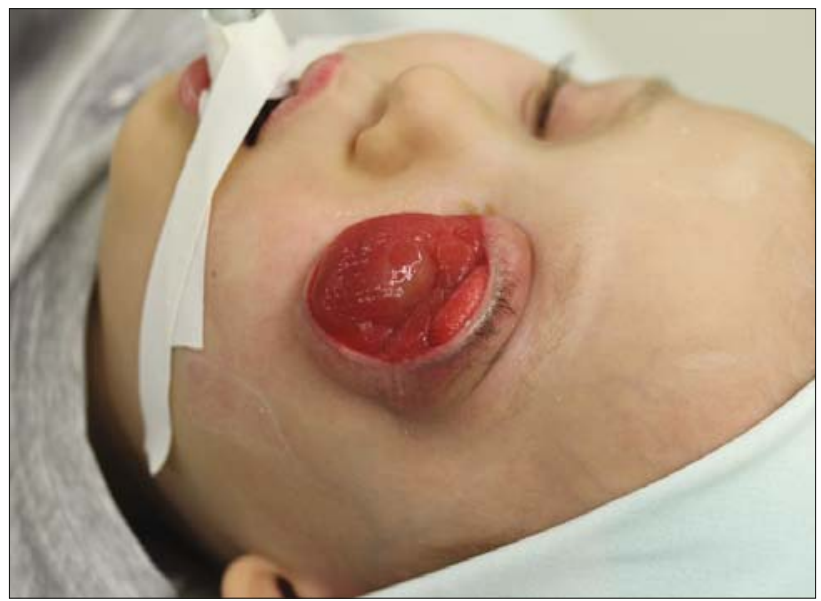

Fig. 5. Patient D.S., February 2017, tumor reduction after 6 courses of CHT, before surgery.

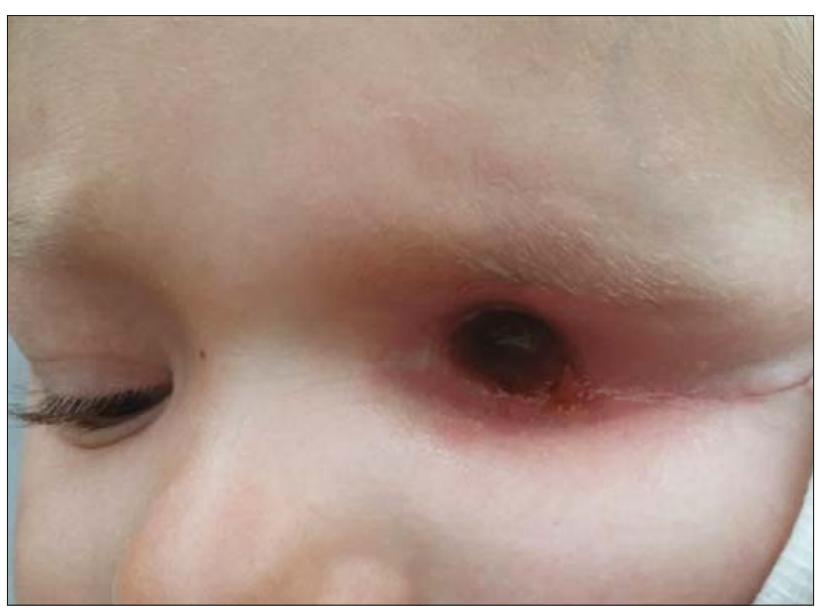

Fig. 6. Patient D.S., April 2017, after surgery (orbital exenteration).

2016 with excessive extraorbital propagation of retinoblastoma (Fig. 2). There was a solid tumor $10.3 \times 7.2 \times 6.2 \mathrm{~cm}$ in left orbital region with spontaneous superficial bleeding, infiltration of regional lymphatic nodes and infiltration of big neck vessels. We diagnosed stage III/b according to International retinoblastoma staging system (IRSS). The extension of retinoblastoma is shown on MRI (Fig. 3). The oncological screening (i. e. cerebrospinal fluid analysis, bone marrow aspiration/biopsy, ultrasound of parenchymatous organs and MRI of CNS) for metastatic disease was negative. Genetic test for RB1 gene mutation was negative. Patient received six courses of chemotherapy with vincristine, etoposide, and carboplatin. After this treatment important improvement of local finding has occurred. The tumor diminished to $60 \times 42 \times 37$ $\mathrm{mm}$ and infiltration of regional lymphatic nodes and infiltration of big neck vessels disappeared (Figs 4 and 5). Patient underwent surgery in February 2017: exenteration of left orbit and lids, with resection of superior and lateral orbital bone wall. Patient received two more courses of chemotherapy (vincristine, etoposide, and carboplatin). 


\section{Results}

Histological examination showed retinoblastoma with massive signs of tumor necrosis. In exenterated mass nods of retinoblastoma cells with blastic morphology were present. Flexner-Winterstein rosettes weren't found in the nods. Margins of resection were tumor free. Genetic examination for RB1 gene mutation was negative. Patient underwent 8 courses of chemotherapy ( 6 before surgery and 2 after surgery- exenteration of left orbit and lids, with resection of superior and lateral orbital wall - disease free (Fig. 6). The last examination in august 2017 including PET, six months after surgery, showed no signs of metastatic disease or local recurrence.

\section{Discussion}

The factors that predicted poor prognosis are: age at treatment initiation, long lag period and extraorbital manifestation. The survival rate in cases of extraorbital propagation of retinoblastoma is low. Cozza et al (1) had in Rome 6 patients with metastatic disease during a 20 year period. Two of them had metastatic disease at diagnosis, 1 patient had trilateral retinoblastoma, 2 children had distant metastatic disease after enucleating and 1 has developed metastatic during conservative treatment. They were treated by chemotherapy and EBRT. Four patients died of progressive disease within 2 years of being diagnosed, two patients alive; 60 and 63 months after diagnosis. (1) In low income countries extraocular and extraorbital retinoblastoma is spreading more often. (6) This higher incidence is due to a delayed referral and treatment. Chawla (6) presents intraocular disease in $72.3 \%$ and extraocular in 27.7 $\%$ of cases in a group of 600 children with retinoblastoma. Metastasis to the central nervous system was noted in $15.7 \%$ of extraocular cases. Authors indicate, that age at diagnosis $>2$ years, lag period $>6$ months and extraocular stage were associated with poor outcome. Extraocular invasion was predictive of low survival (6). The lag interval is a very important prognostic factor. Treatment delay less than 6 months is no longer associated with poor outcomes (7). In UK median referral time from first symptoms to diagnosis by a retinoblastoma specialist was 38 days (7). In India in a large group of 600 patients in extraocular cases the lag period was 7 months (6). In our case it was 46 months. Orbital and loco-regional metastases are curable with intensive chemotherapy, surgery and local radiotherapy $(4,5)$. CNS involvement remains a major factor for poor survival prognosis $(1,3,4,5)$. In series in India, 28 cases of extraocular retinoblastoma were found in stage
III according to IRSS (2). Twenty four out of 28 cases had IRSS stage IIIa and 4 had IRSS stage IIIb retinoblastoma. They used three courses of neoadjuvant chemotherapy, consisting of vincristine, carboplatin and etoposide, followed by enucleation and external beam radiotherapy (EBRT) for the involved orbits and adjuvant VEC chemotherapy for nine more cycles. From 28 patients 16 died, 5 patients were lost to follow up and 7 survived. In our case there were three factors of poor prognosis. Age at treatment initiation was more than 2 years (55 months in our case), extraordinary long lag period (46 months) and extraorbital propagation (stage III/b). We applied chemotherapy in preoperative and postoperative period and surgical therapy. We avoided EBRT because of histological finding of tumor free resection margins and to prevent secondary malignancies in irradiated area. Due to intensive combined treatment the patient is now without signs of metastatic disease or local recurrence.

\section{Conclusions}

We suppose that good treatment results, in spite of the extraordinary long lag interval and a hopeless pretreatment condition, were due to intensive combined treatment in therapeutic naïve retinoblastoma with an absence of RB1 gene mutation.

\section{References}

1. Cozza R, De Ioris MA, Ilari I, et al. Metastatic retinoblastoma: single institution experience over two decades. Brit J Ophthalmol 2009; 93 : 1163-1166.

2. Radhakrishnan V, Sharma S, Vishnubhatla S, Bakhshi S. MRI findings at baseline and after neoadjuvant chemotherapy in orbital retinoblastoma (IRSS stage III). Brit J Ophthalmol 2013; 97 (1): 52-58.

3. Chantada GL, Sampor C, Bosaleh A, Solernou V, Fandiño A, de Dávila MTG. Comparison of Staging Systems for Extraocular Retinoblastoma Analysis of 533 Patients. JAMA Ophthalmol 2013; 131 (9): 1127-1134.

4. PDQ ${ }^{\circledR}$ Pediatric Treatment Editorial Board. PDQ Retinoblastoma Treatment. Bethesda, MD: National Cancer Institute. Updated <06/21/2017.

5. Chawla B, Singh R. Recent advances and challenges in the management of retinoblastoma. Indian J Ophthalmol 2017; 65 (2): 133-139.

6. Chawla B, Hasan F, Azad R et al. Clinical presentation and survival of retinoblastoma in Indian children. Brit J Ophthalmol 2016; 100: 172-178.

7. Posner M, Jaulim A, Vasalaki M et al. Lag time for retinoblastoma in the UK revisited: a retrospective analysis. BMJ Open 2017; 7: e015625. 